\title{
Pathways to polar adaptation in fishes revealed by long-read sequencing
}

Scott Hotaling ${ }^{1}$, Thomas Desvignes ${ }^{2}$, John S. Sproul ${ }^{3}$, Luana S.F. Lins ${ }^{4}$, and Joanna L. Kelley ${ }^{1}$

Affiliations:

${ }^{1}$ School of Biological Sciences, Washington State University, Pullman, WA, USA

${ }^{2}$ Institute of Neuroscience, University of Oregon, Eugene, OR, USA

${ }^{3}$ Department of Biology, University of Nebraska Omaha, Omaha, NE, USA

${ }^{4}$ CSIRO, New South Wales, Canberra, Australia

\section{Correspondence:}

Scott Hotaling, School of Biological Sciences, Washington State University, Pullman, WA, 99164, USA; Email: scott.hotaling@wsu.edu; Phone: (828) 507-9950; ORCID: 0000-0002-59650986

Joanna L. Kelley, School of Biological Sciences, Washington State University, Pullman, WA, 99164, USA; Email: joanna.I.kelley@wsu.edu; Phone: (509) 335-0037; ORCID: 0000-0002$7731-605 X$

Running head: Long-read sequencing of a polar fish

Keywords: polar fish, genome biology, extremophile, Southern Ocean, cold adaptation, antifreeze proteins

\begin{abstract}
:
Long-read sequencing is driving a new reality for genome science where highly contiguous assemblies can be produced efficiently with modest resources. Genome assemblies from longread sequencing are particularly exciting for understanding the evolution of complex genomic regions that are often difficult to assemble. In this study, we leveraged long-read sequencing to generate a high-quality genome assembly for an Antarctic eelpout, Opthalmolycus amberensis, the first for the globally distributed family Zoarcidae. We used this assembly to understand how $O$. amberensis has adapted to the harsh Southern Ocean and compared it to another group of Antarctic fishes: the notothenioids. We showed that from a genome-wide perspective, selection has largely acted on different targets in eelpouts relative to notothenioids. However, we did find some overlap; in both groups, selection has acted on genes involved in membrane structure and DNA repair. We found evidence for historical shifts of transposable element activity in $O$. amberensis and other polar fishes, perhaps reflecting a response to environmental change. We were specifically interested in the evolution of two complex genomic regions known to underlie key adaptations to polar seas: hemoglobin and antifreeze proteins (AFPs). We observed unique evolution of the hemoglobin MN cluster in eelpouts and related fishes in the suborder Zoarcoidei relative to other teleosts. For AFPs, we identified the first species in the suborder with no evidence of afp/ll sequences (Cebidichthys violaceus), potentially reflecting a lineage-specific loss of this gene cluster. Beyond polar fishes, our results highlight the power of long-read sequencing to understand genome evolution.

\section{Introduction:}

Long-reads are revolutionizing genome sequencing and allowing for improved resolution of genotypes underlying phenotypes across the tree of life (Hotaling, Kelley, \& Frandsen, in press; Hotaling, Sproul, et al., 2021). This new potential is particularly valuable for understanding phenotypes that stem from complex, difficult to assemble regions of the genome (e.g., highly repetitive regions). For instance, the evolution of antifreeze proteins (AFPs) - proteins that
\end{abstract}


adsorb to ice and limit its growth (Davies, Baardsnes, Kuiper, \& Walker, 2002; X. Li et al., 1985) - has allowed a wide array of teleost fishes to thrive in marine habitats that are commonly below freezing and ice-laden (Hobbs, Hall, Graham, Davies, \& Fletcher, 2020). AFPs are commonly found in highly repetitive, tandem arrays in the genome and have experienced a large amount of duplication, rearrangement, and modification (e.g., Deng, Cheng, $\mathrm{Ye}, \mathrm{He}$, \& Chen, 2010; Scott, Hew, \& Davies, 1985) Thus, the evolution of AFPs and phenotypes with similarly complex genomic underpinnings has been difficult to understand despite their key role in shaping ecological communities worldwide.

Extreme environments provide insight into the nature of adaptive evolution due to their strong, and often long-term, selective pressures (Kim et al., 2019; S. Xu, Wang, Guo, He, \& Shi, 2020). Extreme habitats are an even more powerful tool when distantly related lineages are subjected to the same selective pressures and their responses are compared. Under this framework, it is possible to disentangle lineage-specific evolutionary responses from more general patterns (e.g., convergent evolution). The Southern Ocean surrounding Antarctica is one of the most contiguous extreme environments on Earth. It is generally demarcated by latitudes south of the Antarctic Convergence (also known as Antarctic Polar Front), where extremely cold (sea surface temperature $<0^{\circ} \mathrm{C}$ ), northward-flowing water from Antarctica meets the comparatively warmer waters of the subantarctic (sea surface temperature $>5.5^{\circ} \mathrm{C}$; Moore, Abbott, \& Richman, 1999). The extreme nature of the Southern Ocean largely stems from three factors: chronic, subfreezing temperatures, high levels of dissolved oxygen, and dramatic seasonal shifts in light availability (DeVries \& Steffensen, 2005). Such extreme conditions greatly influence the biological diversity of the Southern Ocean (Griffiths, 2010) and have driven the evolution of an array of extreme phenotypes (e.g., sea spider gigantism, A. L. Moran \& Woods, 2012).

Among fishes, the most well-known outcome of the long-term selection at both poles is the evolution of AFPs. Based on protein structure, multiple different AFP types have evolved in fishes, including antifreeze glycoproteins (AFGPs) in cod and Antarctic notothenioids and Type III antifreeze proteins in eelpouts (Davies et al., 2002; X. Li et al., 1985). AFPs appear ubiquitous in polar fishes but understanding of their molecular evolution remains incomplete (but see Chen, DeVries, \& Cheng, 1997a; Chen, DeVries, \& Cheng, 1997b; Deng et al., 2010; Zhuang, Yang, Murphy, \& Cheng, 2019). Another peculiarity of life in the Southern Ocean are the white-blooded Antarctic icefishes (family Channichthyidae, Ruud, 1954), which are the only vertebrates known to live without hemoglobin (Beck et al., 2021; Kim et al., 2019). To date, practical limitations have inhibited our understanding of AFP and hemoglobin evolution, particularly the challenge of assembling these repeat-rich, complex loci with short-read sequence data (e.g., Ahn et al., 2017).

Thus far, genomic investigation of Antarctic fishes has been restricted to the notothenioid adaptive radiation (e.g., Kim et al., 2019; Near et al., 2012). These studies have yielded highresolution understanding of how cryonotothenioids (Antarctic notothenioids) have adapted to the Southern Ocean (Chen et al., 2019) and how the unique white-blooded icefishes evolved following the loss of hemoglobin (Near, Parker, \& Detrich III, 2006). Still, many questions remain. Namely, how generalizable is the evolutionary trajectory of cryonotothenioids relative to other fishes in the Southern Ocean? Eelpouts (family Zoarcidae) are common fishes in the Southern Ocean. With roughly $\sim 300$ described species distributed around the world and many in Antarctica (Anderson, 1994; Hotaling, Borowiec, Lins, Desvignes, \& Kelley, 2021; Møller, Nielsen, \& Anderson, 2005), eelpouts are the most speciose family within the suborder Zoarcoidei (also referred to as the infraorder Zoarcales). Similar to cryonotothenioids, eelpouts have evolved AFPs to mitigate freezing stress, specifically Type III AFPs (afplll; X. Li et al., 
1985). The most recent common ancestor of eelpouts and cryonotothenioids diverged $\sim 80$ million years ago (Rabosky et al., 2018), making these groups an ideal system for studying potential convergent evolution to the harsh Southern Ocean.

In this study, we used long-read sequencing to generate a high-quality genome assembly for an Antarctic eelpout, Ophthalmolycus amberensis, the first for the family Zoarcidae. We then leveraged our new genomic resource to better understand how fishes have adapted to the Southern Ocean through whole-genome perspectives on genome evolution and fine-scale investigation of synteny and copy number variation in key adaptive regions (i.e., hemoglobin and AFPs). Collectively, our results highlight convergent and species-specific mechanisms underlying the adaptation of fishes to the harsh conditions of the Southern Ocean. Our study also provides practical evidence for the power and promise of long-read sequencing for understanding genome biology, particularly in highly repetitive and/or complex genomic regions.
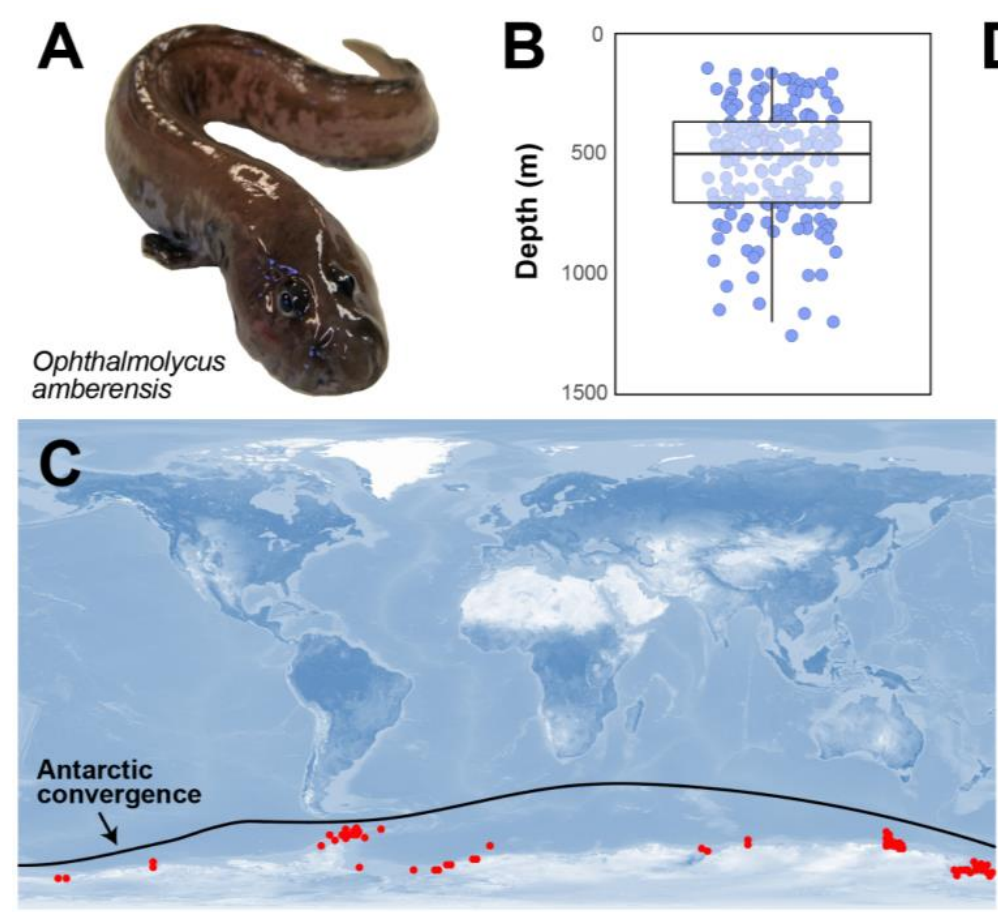

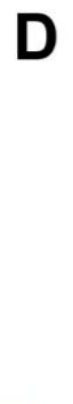

\begin{tabular}{l|l}
\hline \multicolumn{1}{l}{ Assembly } & \multicolumn{1}{l}{ O. amberensis } \\
\hline Total length & $679.26 \mathrm{Mb}$ \\
$\begin{array}{l}\text { No. of scaffolds } \\
\text { Longest scaffold }\end{array}$ & 1,828 \\
$6.97 \mathrm{Mb}$ \\
$\begin{array}{l}\text { Scaffold N50 } \\
\% \mathrm{~N}, \text { assembly }\end{array}$ & $1.00 \mathrm{Mb}$ \\
\hline BUSCO & \multicolumn{2}{|l}{} \\
\hline Complete, all & $4,420(96.4 \%)$ \\
$\begin{array}{l}\text { Complete, } \\
\text { single copy }\end{array}$ & $4,237(92.4 \%)$ \\
$\begin{array}{l}\text { Complete, } \\
\text { duplicated }\end{array}$ & $183(4.0 \%)$ \\
$\begin{array}{l}\text { Fragmented } \\
\text { Missing }\end{array}$ & $86(1.9 \%)$ \\
$\begin{array}{l}\text { Total BUSCOs } \\
\text { searched }\end{array}$ & $78(1.7 \%)$ \\
$\begin{array}{l}\text { Reference } \\
\text { data set }\end{array}$ & 4,584 \\
\hline
\end{tabular}

Figure 1. (A) An Antarctic eelpout, Ophthalmolycus amberensis, sampled from the Gerlache Strait on the West Antarctic Peninsula. (B) Depth records ( $n=168$ records) for O. amberensis from GBIF (https://www.gbif.org/). (C) The distribution of O. amberensis from AquaMaps

(https://www.aquamaps.org/). Red circles represent sampling localities and the dark line indicates the Antarctic Convergence (or Antarctic Polar Front) where subfreezing northward flowing Antarctic waters meet the comparatively warmer waters of the sub-Antarctic. Sea surface temperatures south of the Antarctic Convergence are commonly below $0^{\circ} \mathrm{C}$. (D) Assembly and BUSCO (Benchmarking Universal Single-Copy Orthologs; Simão, Waterhouse, loannidis, Kriventseva, \& Zdobnov, 2015) for the $O$. amberensis genome assembly presented in this study.

\section{Materials and methods:}

Species description and sample collection Ophthalmolycus amberensis is a coastal Antarctic species, likely with a circumpolar distribution (Fig. 1). Ophthalmolycus amberensis individuals were collected from 360-380 m in May 2016 using a 5-feet Blake trawl deployed from the Antarctic Research and Supply Vessel (ARSV) 
Laurence M. Gould in the Gerlache Strait $\left(64^{\circ} 44^{\prime} \mathrm{S}, 63^{\circ} 01^{\prime} \mathrm{W}\right)$ on the West Antarctic Peninsula. Live fish were transferred immediately from the trawl net to the ship aquaria for transport to the aquatic facilities at Palmer Station, Antarctica, where they were maintained in flow-through seawater tanks at $\sim 0^{\circ} \mathrm{C}$. Following euthanasia with a lethal dose of MS-222 (Syndel, Ferndale, WA, USA), individuals were dissected and sampled with skeletal muscle flash frozen in liquid nitrogen and stored at $-80^{\circ} \mathrm{C}$, fin clips stored in $80 \%$ ethanol at room temperature, and liver preserved in RNALater until it could be moved to longer term $-80^{\circ} \mathrm{C}$ storage. All procedures were performed according to protocols approved by the Institutional Animal Care and Use Committees (IACUC) of the University of Oregon (\#13-27RRAA). We confirmed visual species identification through sequencing of the mitochondrial cytochrome c oxidase I ( $m t$-co1) locus and comparison to sequences on GenBank.

146

\section{Sequence data collection}

We collected three sequence data sets for this study: Iong-read Pacific Bioscience (PacBio) data, short-read Illumina whole-genome (WGS) data, and short-read Illumina transcriptomic (RNA-seq) data. Our sequence data were collected from three individuals and tissue types: PacBio (EP06, muscle), WGS (EP10, fin clip), and RNA-seq (EP01, liver). PacBio Sequel SMRTbell libraries were prepared and sequenced at the Oregon State University Center for Quantitative Life Sciences on a PacBio Sequel platform with eight SMRT cells. For WGS library preparation, genomic DNA was extracted using a MagAttract HMW DNA kit and the library was prepared following an Illumina PCR-free protocol with a 400-bp insert size. The resulting WGS library was sequenced at MedGenome, Inc. on an Illumina HiSeq 4000 platform with 150-bp paired-end sequencing chemistry. Total RNA was extracted from $~ 30 \mathrm{mg}$ of liver tissue using the Macherey-Nagel NucleoSpin $\AA$ kit and the manufacturer protocol. The tissue was flash frozen with liquid nitrogen and crushed to homogenize it before extraction. An RNA-seq library was prepared by the University of Utah Genomics Core Facility and sequenced on an Illumina HiSeq 2500 with 125-bp paired-end sequencing chemistry. We assessed sequence quality for both short-read data sets (WGS and RNA-seq) with FastQC v0.11.4 (Andrews, 2010). We trimmed the raw WGS reads with Trim Galore! v0.4.2 (Krueger, 2015) with stringency of 5, quality of 28 , and a minimum length of $50 \mathrm{bp}$.

\section{Genome assembly}

We first estimated the genome size and heterozygosity of the O. amberensis genome for the WGS data using the online portal of GenomeScope (Vurture et al., 2017). We produced the input for GenomeScope, a histogram of 21-mer counts, with jellyfish v2.2.5 (Melsted \& Pritchard, 2011). We also estimated genome size from the WGS data with sga preQC v0.10.14 (Simpson \& Durbin, 2010). For genome assembly, we first combined our separate subread BAM files into a combined BAM using 'pbmerge', from the PacBio toolkit. Next, using 'bam2fastx', another PacBio tool, we converted the raw, combined subread BAM into FASTQ format for downstream analyses. Scripts and commands used in this study are provided GitHub (http://github.io/oamberensis genome).

We assembled the genome from PacBio sequences only using Canu v1.8 (Koren et al., 2017), a dedicated long-read assembler designed for PacBio data. We changed one parameter, cnsErrorRate $=0.25$, all other parameters were left as defaults. For all iterations of the genome assembly, we generated summary statistics using the Assemblathon2 summary script (assemblathon_stats.pl, Bradnam et al., 2013). We also assessed completeness by calculating the number of observed single-copy orthologs (BUSCOs) in the assembly using BUSCO v3 and the 4,584 "actinopterygii_odb9" reference gene set (Simão et al., 2015). 
According to our BUSCO results, the initial Canu assembly (v1) of the 0 . amberensis genome was $\sim 40 \%$ larger than expected at $1.02 \mathrm{~Gb}$ with many duplicated regions $(n=1180$ complete and duplicated BUSCOs), likely due to heterozygosity between haplotypes. To resolve duplicate contigs we used Purge Haplotigs (Roach et al. 2018). We first mapped raw PacBio reads to the v1 assembly with Minimap2 v2.14-r883 (Li 2018) and the options: -ax -map-pb. Next, we sorted the resulting BAM file of aligned reads with samtools v1.9 (Li et al. 2009) and default settings. We used the 'readhist' function of Purge Haplotigs to generate a coverage histogram of the mapped PacBio reads. We set low (-I), middle (-m), and high (-h) in the coverage distribution of $10,140,195$, respectively, and ran the second step in the Purge Haplotigs pipeline, 'contigcov', to analyze coverage on a contig-by-contig basis. Finally, we purged duplicate contigs using the function 'purge' to create a de-duplicated assembly. The resulting assembly (v2) was substantially smaller at $679 \mathrm{Mb}$ with $84.5 \%$ fewer duplicated genes.

We then polished the v2 assembly using our raw PacBio reads with Arrow, an algorithm in the variantCaller program within the Falcon software (Chin et al. 2013, 2016). Since this approach is not deterministic (i.e., repeated runs will always find changes), we performed two rounds of polishing with Arrow (resulting in assemblies v3 and v4). For each round, we first re-mapped our raw $\mathrm{PacBio}$ reads to the latest assembly version (e.g., to make $\mathrm{v} 3$, we mapped raw PacBio reads to the v2 assembly) with Minimap2 v2.14-r883 ( $\mathrm{Li} \mathrm{2018)}$ and the same flags as above. Next, we converted the BAM file of mapped reads to PacBio format with 'pbbamify' (part of the PacBio toolkit) with default flags. We sorted the re-formatted BAM file with samtools v1.9 as above (Li et al. 2009). We created an index of the mapped, sorted BAM with another PacBio tool, 'pbindex'. This process was used to create the polished v3 assembly and repeated for v4.

\section{Transcriptome assembly}

To aid in genome annotation, we assembled an $O$. amberensis liver transcriptome from raw RNA-seq reads with Trinity v2.6.6 (Grabherr et al. 2011) using default settings and read trimming with Trimmomatic (Bolger et al. 2014) with flag "--trimmomatic." We assessed transcriptome assembly completeness with BUSCO v3 and the 4,584 "actinopterygii_odb9" reference gene set (Simao et al. 2015).

\section{Genome annotation}

We annotated the $O$. amberensis genome assembly (v4) using Maker2 v31.10 (Holt \& Yandell, 2011). To support the annotation pipeline, we generated a repeat library using RepeatModeler v1.0.11 with default settings (A. F. Smit \& Hubley, 2008). We also included gene evidence from the $O$. amberensis transcriptome and proteins for two other species, three-spined stickleback (Gasterosteus aculeatus) and Atlantic cod (Gadus morhua), which were both downloaded from Ensembl release 97 (http://ftp.ensembl.org/pub/release-97/). We first performed a de novo annotation of the $O$. amberensis genome assembly with our transcriptome and protein evidence used with options est2genome $=1$ and protein2genome $=1$ in the Maker2 control file. Next, we used the gene finding program, SNAP (Korf, 2004), to generate ab initio gene predictions for our assembly. We first merged all of our gene models from our de novo Maker2 together into a single GFF3 using the Maker2 tool "gff3_merge" and converted them to ZFF format with "maker2zff." We trained SNAP on these data by first breaking up our sequences into one gene per locus (with 1000 bp on either side of the predicted gene) using "fathom" (part of SNAP). We estimated parameters for our genes using "forge" (also included with SNAP) before building a Hidden Markov Model for our annotations with the script "hmm-assembler.pl". After SNAP training, we re-ran Maker2 with our trained SNAP models by adding them in the control file ("snaphmm" option) and setting est2genome and protein2genome both to 0 . We performed a second round of SNAP training using the same approach followed by running Maker2 for a third time. Next, we prepared to train another gene predictor, Augustus v2.5.5 (Stanke \& Waack, 
2003), on our gene models by first merging the gene models produced in the second round the SNAP training using "gff3_merge", converting them to ZFF with "maker2zff", and then to GBK format with a custom perl script (https://github.com/hyphaltip/genomescripts/blob/master/gene prediction/zff2augustus gbk.pl). As Augustus is a machine learning approach, we first split our data set into a training and test set using the script "randomSplit.pl." We then created a new "species" for Augustus with the script "new_species.pl" then performed training by running the "etraining" function on our training data set followed by evaluating its performance with the "augustus" tool. We optimized our prediction parameters with the script "optimize_augustus.pl." After Augustus training, we re-ran Maker2 as above with the addition of the control file option "augustus_species." Finally, we ran GeneMark for the O. amberensis genome assembly with the self-training algorithm using flag -ES. We then performed a final run of Maker2 with all of the gene model evidence included by adding the path to our GeneMark trained predictions using "gmhmm" to our control file along with the above evidence for Augustus ("augustus_species") and SNAP ("snaphmm"). We kept est2genome and protein2genome as 0 for this final run.

\section{Repetitive element classification and annotation}

We identified and classified repetitive elements (REs) in the genome assembly of $O$. amberensis using RepeatModeler v2.0.1 (Flynn et al., 2020). We then used the "queryRepeatDatabase.pl" script included with RepeatMasker v4.1.0 (A. Smit, Hubley, \& Green, 2015) to output RepeatMasker's internal library for ray-finned fishes (-species "actinopterygii") and merged it with the $O$. amberensis custom repeat library generated by RepeatModeler. We used this combined Actinopterygii $+O$. amberensis library to annotate REs in the $O$. amberensis assembly using RepeatMasker v4.1.0 with the search engine set to "ncbi" and the -xsmall option. We repeated the above steps to classify and annotate REs in each of five additional fish genome assemblies-Gasterosteus aculeatus (BROAD S1, Ensembl release 97), Gadus morhua (gadMor1, Ensembl release 97), Takifugu rubripes (FUGU5, Ensembl release 98), Chaenocephalus aceratus (Chaenocephalus aceratus V1.0), and Parachaenicthys charcoti (Parachaenichthys charcoti V1.0, GigaDB dataset \#100321) — such that each assembly was annotated using a combined library of Actinopterygii + the custom library from RepeatModeler for each species. We summarized repeat abundance by parsing output from RepeatMasker and generating plots in R v3.5.1 (R Core Team, 2021) using custom scripts. To investigate patterns of transposable element (TE) activity through time we generated TE copy number divergence plots. We calculated Kimura substitution level (CpG adjusted) for transposable elements using the RepeatMasker utility script "calcDivergenceFromAlign.pl", removed non-transposable element and unclassified repeats from the resulting ".divisum" file, and generated age distribution landscapes using RepeatMasker's "createRepeatLandscape.pl" script.

\section{Selection on protein-coding genes}

To assess selection on protein-coding genes in the $O$. amberensis genome, we calculated the ratio of non-synonymous to synonymous substitution $(d N / d S)$ using CodeML with the PAML package (Yang, 2007). We first identified orthologous groups of single-copy coding sequences using the OrthoVenn2 web server (L. Xu et al., 2019). Coding sequences for the same six fish genomes as the repeat analyses above were included: G. aculeatus, G. morhua, $O$. amberensis, $T$. rubripes, $C$. aceratus, and $P$. charcoti. To identify orthologous gene groups, we translated coding sequences (CDS) to protein using the function "transeq" in EMBOSS v.6.6.0 (Rice, Longden, \& Bleasby, 2000). We then extracted corresponding CDS for each group of single-copy orthologs for each taxon. We aligned each of the orthologous CDS with PRANK v.170427 (Löytynoja, 2014) under a codon model. Before performing $d N / d S$ analyses, we generated a species tree with RAxML v.8.2.12 (Stamatakis, 2014) for a concatenated alignment 
286

287

288

289

290

291

292

293

294

295

296

297

298

299

300

301

302

303

304

305

306

307

308

309

310

311

312

313

314

315

316

317

318

319

320

321

322

323

324

325

326

327

328

329

330

331

332

333

334

335

336

of all of the single-copy orthologous nucleotide sequences identified above with 100 bootstrap replicates using a GTR+Gamma model.

Using CodeML, we calculated $d N / d S$ for aligned single-copy orthologous coding sequences with branch-site models A1 (model $=2$, NSsites $=2$, fix_omega $=1$ ) and A $($ model $=2$, NSsites $=2$, fix_omega $=0$ ) and F $3 X 4$ codon frequency and cleandata $=1$ for both. Using the same models, we varied the foreground lineage(s) by first focusing only on $O$. amberensis with all other lineages as background and second with the two notothenioids $(C$. aceratus and $P$. charcoti) as foreground lineages. To statistically compare our test model (A) to our null neutral model (A1) for each group of orthologs, we performed a Chi-Square Test. To obtain $P$-values for all chisquare values, we used the pchisq() function in $R$ v.3.6.3 (R Core Team, 2021). To control for multiple tests, we performed false discovery rate (FDR) correction in R using the p.adjust() function and the "Benjamini-Hochberg" method (Benjamini \& Hochberg, 1995) with a cutoff of 0.05 . We identified Gene Ontology (GO) terms for genes that exhibited evidence of positive selection using Ensembl IDs for G. aculeatus and tested for GO term enrichment among target lists using ShinyGO v0.74 (Ge, Jung, \& Yao, 2020). For GO term enrichment analyses, we compared lists of genes with evidence of positive selection against the background of all singlecopy orthologs. We assessed GO term enrichment for three categories of terms: biological process, cellular component, and molecular function.

\section{Annotating hemoglobin and AFP regions}

To explore the evolution of hemoglobin genes in $O$. amberensis and across the suborder Zoarcoidei, hemoglobin gene clusters were analyzed and compared to other species representing major perciformes lineages: Cebidichthys violaceus (Cvio_1.0, Heras, Chakraborty, Emerson, \& German, 2020), Anarrhichthys ocellatus (GSC̄_Weel_1.0), P. gunnellus (fPhoGun1.1), Perca flavescens (PFLA_1.0, Feron et al., 2020), Etheostoma spectabile (UIUC_Espe_1.0, R. L. Moran, Catchen, \& Fuller, 2020), G. aculeatus (BROAD S1), Pseudoliparis sp. (ASM433547v1, Mu et al., 2021), Cottoperca gobio (fCotGob3.1, Bista et al., 2020), Ophiodon elongatus (NWFSC_Oelon_r3.mflye, Longo et al., 2020), Cyclopterus lumpus (fCycLum1.pri), and Taurus bubalis (fTauBub2.1). In teleost fish, hemoglobin genes occur in two distinct, unlinked clusters (LA and MN) which were first located in each assembly by searching for flanking genes (i.e., rhbdf1b and aqp8.2 for the LA cluster and kank2 and nprl3 for the MN cluster). Sequence, length, position, and strand of individual exons of each alpha and beta gene were manually retrieved from each assembly by performing BLASTN searches and using the yellow perch $P$. flavescens as a reference for hemoglobin gene exon boundaries. Exons of each gene were concatenated and translated into protein sequence to verify their accuracy. If a gene was incomplete (e.g., missing an exon) or displaying a premature stop codon, the gene was considered pseudogenized.

To explore the evolution of AFPs across the Zoarcoidei (and an outgroup, G. aculeatus), region associated with afp/ll genes and their progenitor in eelpouts, sialic acid synthase (nans; Deng et al., 2010), were annotated by hand via a combination of BLASTN searches and focused alignments. We focused on six species (five from Zoarcoidei) with high-quality genomic resources-five genome assemblies [G. aculeatus (BROAD S1), O. amberensis, C. violaceous (Cvio_1.0), A. ocellatus (GSCB_Weel_1.0), P. gunnellus (fPhoGun1.1)] and one set of bacterial artificial chromosomes (BACs) which targeted the same regions in another Antarctic eelpout, Lycodicthys dearborni (Deng et al., 2010). For all genes except afplll, we used all of the known exons for G. aculeatus from Ensembl 104. For afplll genes, we used a combination of the two exons described by Deng et al. (2010) for L. dearborni and Hobbs et al. (2020) for P. gunnellus. We first performed a BLASTN v.2.2.31 search with default settings against a reference database (created with "makeblastdb" for each genome or BAC group) and each exon as a 
separate query. We further investigated regions of interest by extracting them with samtools $(\mathrm{H}$. $\mathrm{Li}$ et al., 2009) using the function "faidx" and aligning sequences with Clustal Omega (Sievers et al., 2011).

Where appropriate, we assessed the quality of the assembly at target regions within the hemoglobin and AFP loci by mapping whole-genome, short-read lllumina data from a different $O$. amberensis individual to the $O$. amberensis PacBio genome assembly using bwa v.0.7.12 (Heng Li, 2013). We indexed the PacBio assembly using the "index" function then mapped reads with the "bwa-mem" algorithm with default settings. We converted the output from a BAM to SAM file with samtools (H. Li et al., 2009) and visualized regions with the Integrated Genomics Viewer (Robinson et al., 2011).

\section{Results:}

Sequence data

We generated 5,536,887 long-read PacBio sequences $(65.7 \mathrm{~Gb}$, mean $=13.2 \mathrm{~Kb}$, read N50 $=$ $20 \mathrm{~kb}$ ) and 237,098,976 Illumina short-reads (WGS = 226,420,980; RNA-seq = 10,677,996). We estimated the $O$. amberensis genome to be $689.8 \mathrm{Mb}$ from the WGS data. Based on this estimate, our sequencing coverage for the PacBio data was $95 x$ and $49 x$ for the WGS data. We estimated the genome heterozygosity to be $0.36 \%$. The genome assembly, raw sequence data, and associated files for O. amberensis are deposited under NCBI BioProject PRJNA701078.

\section{O. amberensis genome assembly and annotation}

Our final assembly of the $O$. amberensis genome ( $\mathrm{v} 4$ ) was $680.7 \mathrm{Mb}$ in 1,828 scaffolds with an $\mathrm{N} 50$ of $1.00 \mathrm{Mb}$. The longest scaffold was $6.98 \mathrm{Mb}$. Based on the $\mathrm{k}$-mer based estimate of genome size, our assembly represents $98.7 \%$ of the genome. The assembly contains $95.7 \%$ of the single-copy, conserved orthologous genes (BUSCOs) in the 4,584 gene reference set for Actinopterygii (Fig. 1D). We annotated 22,572 genes in the $O$. amberensis genome assembly. According to the same BUSCO gene set used for assessing the genome assembly completeness, the $O$. amberensis liver transcriptome used for annotation was $67.1 \%$ complete with $9.1 \%$ of genes fragmented and $23.8 \%$ missing.

Repetitive element content Repetitive elements (REs) comprised $30.8 \%$ (209.6 Mb) of the O. amberensis genome assembly (Fig. 2). Of all repetitive sequences masked by RepeatMasker, 38.6\% (78.8 Mb) were classified as repeats. DNA transposons accounted for the largest fraction of classified REs (33.8Mb or $16.2 \%$ of total REs), followed by long-interspersed nuclear elements (LINEs) (25.2Mb or $12.0 \%$ ) and long-terminal repeats (LTRs; $15.0 \mathrm{Mb}$ or $7.2 \%$ ), respectively. Unclassified REs accounted for the remaining repetitive component $(130.1 \mathrm{Mb}$ or $62.4 \%$ total $\mathrm{REs}$ ) of the $O$. amberensis assembly.

Opthalmolycus amberensis and the two notothenioid species (C. aceratus and $P$. charcoti) showed greater RE abundance in both genomic proportion and total repeats (range $=30.8-$ $49.4 \%$ or $209.6-525.6 \mathrm{Mb}$ in $O$. amberensis and C. aceratus, respectively), relative to the remaining non-Antarctic species (range $=11.9-24.6 \%$ or $52.7-205.0 \mathrm{Mb}$ in T. rubripes and G. morhua, respectively; Fig. 2). Although satellite DNAs make up a small fraction of REs in all species, satellites were at least 10 times more abundant (in terms of genomic proportion) in the $O$. amberensis assembly compared to all other assemblies except $C$. aceratus, for which they were approximately five times more abundant. For all species either DNA transposons or LINEs comprised the largest proportion of classified repeats. 
bioRxiv preprint doi: https://doi.org/10.1101/2021.11.12.468413; this version posted November 12, 2021. The copyright holder for this preprint (which was not certified by peer review) is the author/funder, who has granted bioRxiv a license to display the preprint in perpetuity. It is made available under aCC-BY 4.0 International license.

A RE Genome \% B RE Total Bases

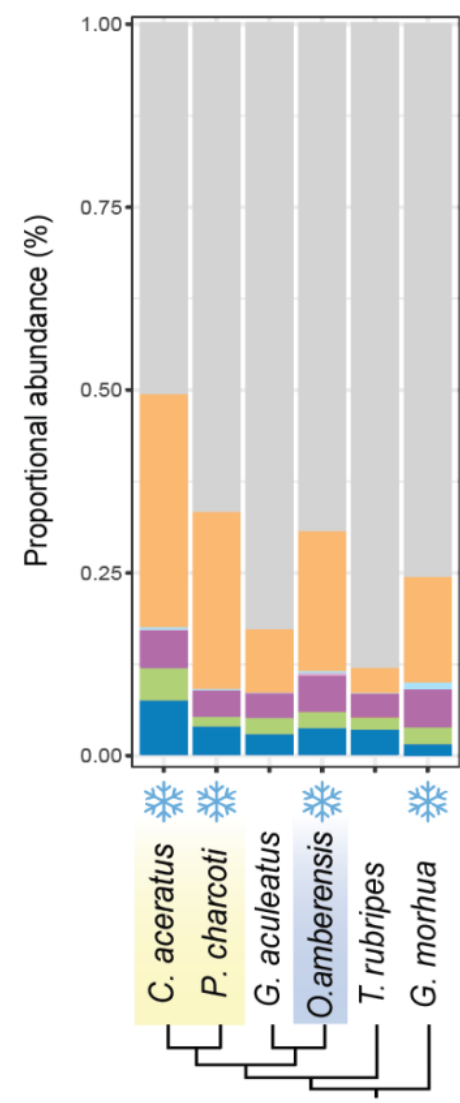

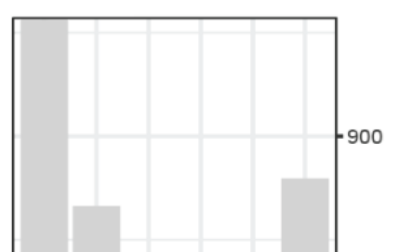
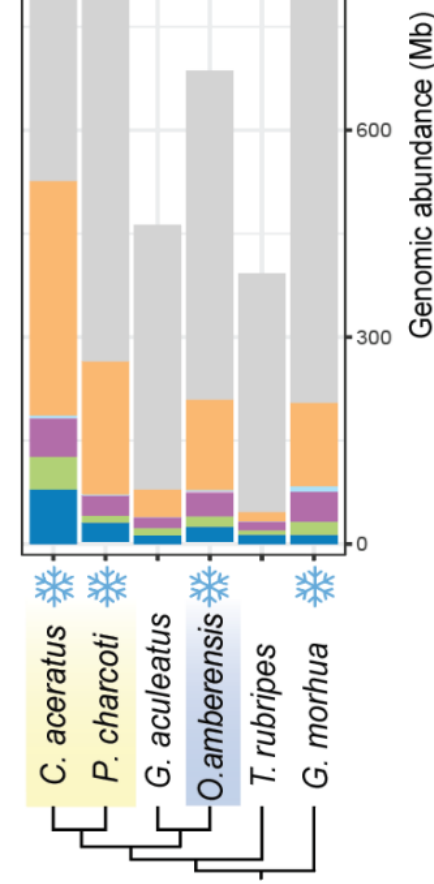

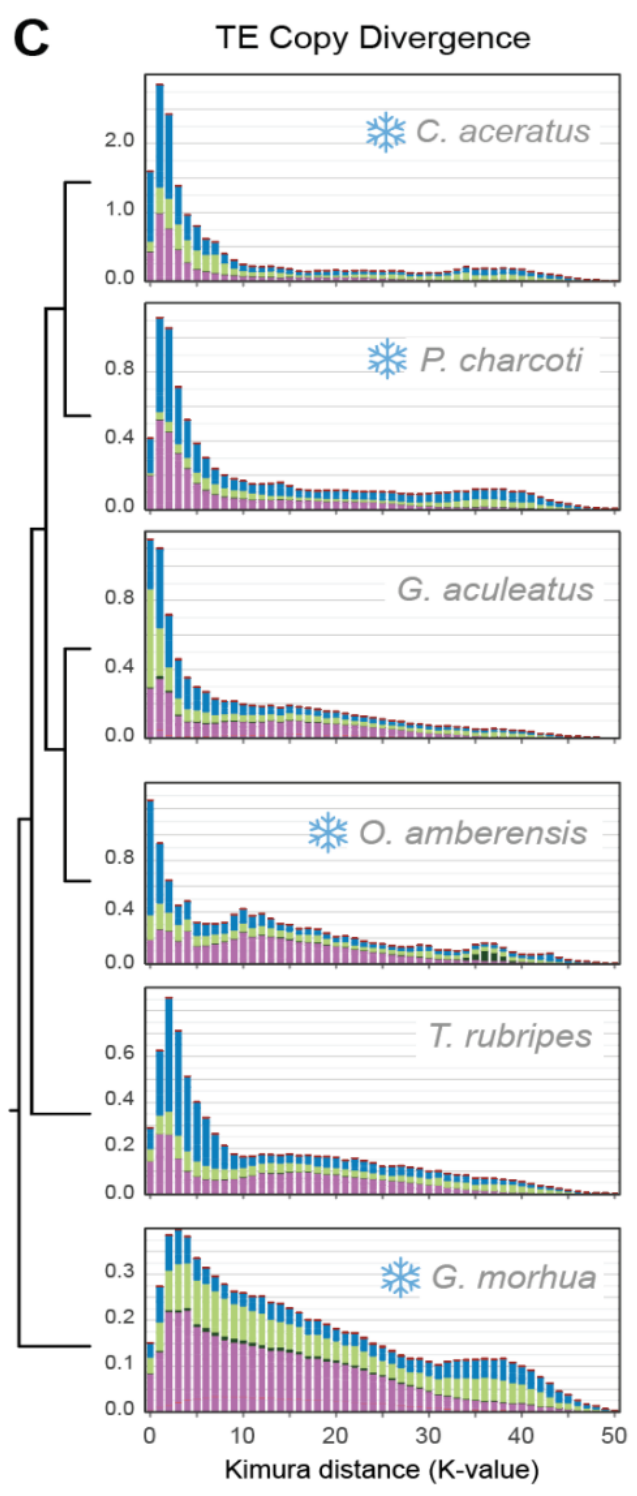

Figure 2. Repetitive element abundance in focal fishes. (A) Abundance of major repetitive element (RE) categories shown as genomic proportions for $O$. amberensis and five other species, $C$. aceratus, $P$. charcoti, G. aculeatus, T. rubripes, and G. morhua. Yellow and blue boxes indicate the Antarctic notothenioids and eelpout shown in Figure 3, respectively. (B) RE abundance shown as total bases in the assembly such that the height of each bar is proportional to the assembly length for that species, with the assembly fraction not masked by RepeatMasker shown in gray. (C) Transposable element (TE) copy divergence plots. The $y$-axis shows TE abundance as a proportion of the genome (e.g., $1.0=1 \%$ of the genome). The $\mathrm{x}$-axis shows sequence divergence ( $\mathrm{CpG}$ adjusted Kimura distance) relative to consensus sequences for TE superfamilies. Copy number peaks with abundance skewed toward the left (i.e., low sequence divergence) may represent TE copies with a recent history of diversification relative to peaks with right-skewed abundance which may comprise remnants of more ancient bursts of TE activity. To simplify visual display, TE superfamilies within DNA transposons, Helitrons, LTRs, LINEs, and SINEs were grouped by the same color. A similar plot with all superfamilies separated by color is shown in Fig. S1. Snowflakes indicate species with distributions that include polar seas.

Transposable element (TE) copy divergence plots in O. amberensis suggested evidence of dynamic shifts in transposable activity through time (Fig. 2). LINEs dominated recent and 
406

407

408

409

410

411

412

413

414

415

416

417

418

419

420

421

422

423

424

425

426

427

428

429

430

431

432

433

434

435

436

437

438

439

440

441

442

443

444

ongoing TE activity driven by recent expansion of $L 2$ and Rex/Babar elements, accounting for much of the LINE abundance (blue peak between $K=0-5$; Fig. 2C, Fig. S1). DNA transposons showed a relatively older history of proliferation dominated by $h A T$ elements (broad peak centered at $K=10$; Fig. 2C, Fig. S1), though some more recent peaks are also evident. Prior to this putative increase in DNA transposon activity, Helitrons showed evidence of an ancient burst with abundant sequences centered over $K=35-40$ (Fig. 2C, Fig. S1). LTRs appear to have maintained modest activity through time, although ERV1 and Gypsy elements show some evidence of recent expansion (Fig. 2C, Fig. S1). Similar trends of recent LINE expansions were visible in the other two Antarctic species, $C$. aceratus and $P$. charcoti, as well as $T$. rubripes. Recent DNA transposon activity was evident in all species, though least pronounced in $O$. amberensis. Gasterosteus aculeatus was the only species for which LTRs dominated recent TE activity (mostly Gypsy, ERV1, and Pao elements; Fig. 2C, Fig. S1). The three other polar species, $C$. aceratus, $P$. charcoti, and $G$. morhua, all have evidence for ancient TE expansions similar to the Helitron expansion in $O$. amberensis, however in these species LINEs and LTRs drove the expansion (Fig. 2C, Fig. S1).

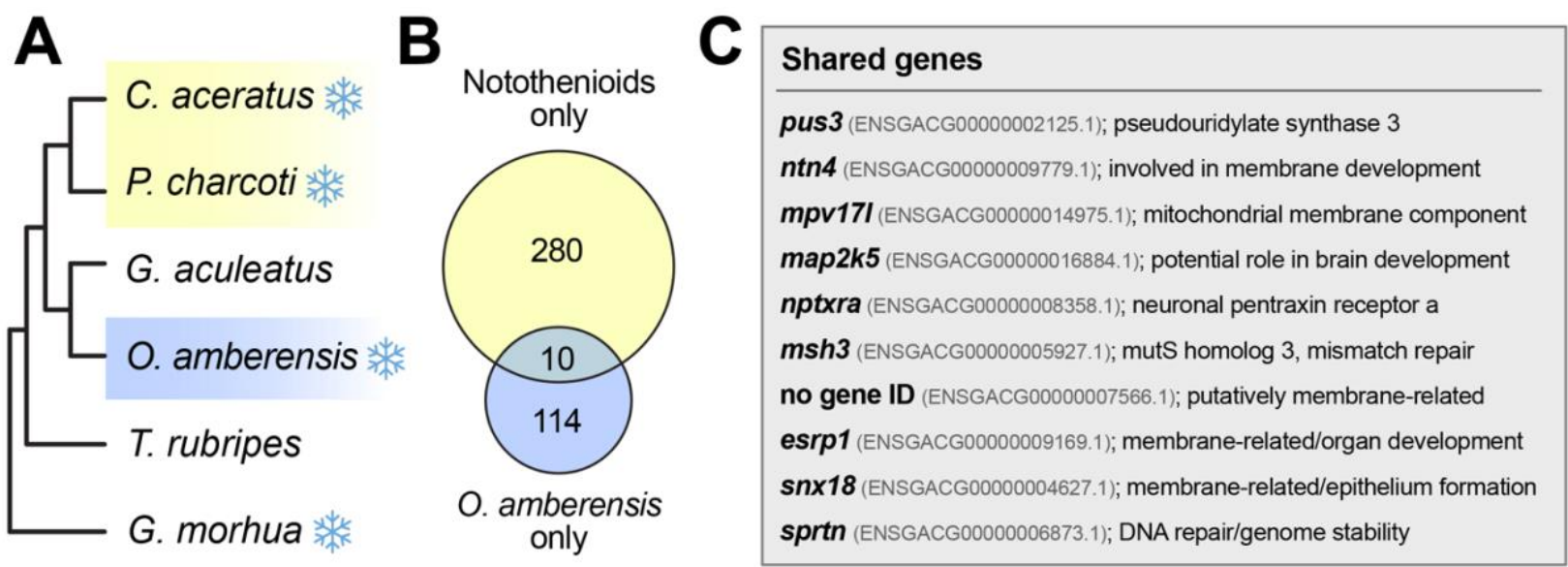

Figure 3. (A) The guide tree generated for $d N / d S$ analyses from 3,461 protein-coding genes. Yellow and blue boxes indicate the foreground lineages in respective analyses (top, yellow: notothenioids only; bottom, blue: $O$. amberensis only). (B) The number of genes with evidence of positive selection in each independent analysis and in both analyses (middle of the Venn diagram). (C) The 10 genes that were shared between both analyses. When applicable, gene names and IDs from the ortholog in stickleback (Gasterosteus aculeatus, BROAD S1) are given. The FDR cutoff used in all analyses was 0.05 .

Snowflakes indicate species with distributions that include polar seas (Antarctic: C. aceratus, P. charcoti, O. amberensis; Arctic: G. morhua).

Selection on protein-coding genes

We identified 3,461 single-copy orthologous genes that were present in all six focal species. When only $O$. amberensis was the foreground lineage in $d N / d S$ analyses, we identified 123 protein-coding genes with evidence of positive selection with FDR $<0.05$ (Fig. 3). When the two notothenioids ( $P$. charcoti and $C$. aceratus) were the foreground lineages, 289 protein-coding genes exhibited evidence of positive selection. When these two lists were intersected, just 10 protein-coding genes exhibited evidence of positive selection in both groups, leaving 113 $(91.9 \%)$ and $279(96.5 \%)$ genes that were uniquely under positive selection in 0 . amberensis or the notothenioids, respectively (Fig. 3). The gene that exhibited the strongest signature of positive selection in O. amberensis (Ensembl ID \#ENSGACG00000014975; FDR = 2.02e-10) is orthologous to human MPV17L, a component of the mitochondrial membrane. For the notothenioids, the strongest signature of positive selection was eif4enif1 (FDR $=5.06 e-30$ ), a 
eukaryotic translation initiation factor which has been linked to thermal stress in salmonids (Akbarzadeh et al., 2018).

For the 10 positively selected genes shared between 0 . amberensis and the two notothenioids, there were not enough genes to assess higher level groupings of GO terms for biological processes. For cellular components, however, several genes (snx18, sprtn, and esrp1) were identified as playing roles in organelle and/or membrane processes. Only one pathway was significantly enriched-Phosphatidylinositol-4,5-bisphosphate binding (FDR $=0.046$ ) -and it included one positively selected gene $(s n \times 18)$. For both the $O$. amberensis and the notothenioid-specific analyses, there was no evidence of significant GO term enrichment. However, here we report the top GO terms associated with positively selected genes in each analysis. For the 124 genes detected in the $O$. amberensis only analysis, the most common GO categories for biological processes were "regulation of biological process" ( $n=26$ genes), "regulation of cellular process" ( $n=24)$, and "response to stimulus" $(n=24)$. For cellular components, the most common GO categories were "organelle" $(n=30)$, "membrane-bounded organelle" $(n=28)$, and "intracellular organelle" $(n=28)$. For molecular function, "protein binding" ( $n=29$ ), "ion binding $(n=27)$, and "organic cyclic compound binding" $(n=25)$ were the most common. Finally, for the 290 genes detected in notothenioids, the most common GO categories were very similar to those of $O$. amberensis. For biological processes, "regulation of biological process" ( $n=63$ ), "regulation of cellular process" $(n=59)$, and "multicellular organismal process" $(n=51)$ were the most common. For cellular components, the most common were "organelle" $(n=83)$, "intracellular organelle" $(n=81)$, and "membrane-bounded organelle" ( $n=75)$. For molecular function, "protein binding" $(n=79)$, "ion binding $(n=66)$, "organic cyclic compound binding" ( $n=66)$, and "heterocyclic compound binding" $(n=66)$ were most common.

\section{Hemoglobin and AFPs}

In $O$. amberensis and across Zoarcoidei, the LA cluster remained stable with the typical organization of two alpha-globin and one beta-globin genes (Opazo et al. 2013). However, among perciformes, the beta-gene of the LA cluster, $h b b l a$, was repeatedly lost or pseudogenized in some species, including in the Zorcoidei monkeyface prickleback Cebidichthys violaceus. In contrast, the MN cluster in perciformes underwent dynamic changes in a lineage specific fashion and harbored up to 13 alpha-globin and 15 beta-globin genes in the Longspined bullhead sculpin Taurulus bubalis and numerous instances of pseudogenization, especially frequent in the Orangethroat darter Etheostoma spectabile. While the MN cluster organization could not be analyzed in the wolf-eel $A$. ocellatus due to the lack of contiguity in the assembly at this locus, within Zoarcoidei, however, the MN cluster composition remained relatively stable among the three studied species with three alpha-globin genes and two or three functional beta-genes. A specificity of Zoarcoidei compared to the other species is the presence of a hbbmn2 pseudogene in $C$. violaceus and $P$. gunnellus and the loss of this gene in $O$. amberensis. 


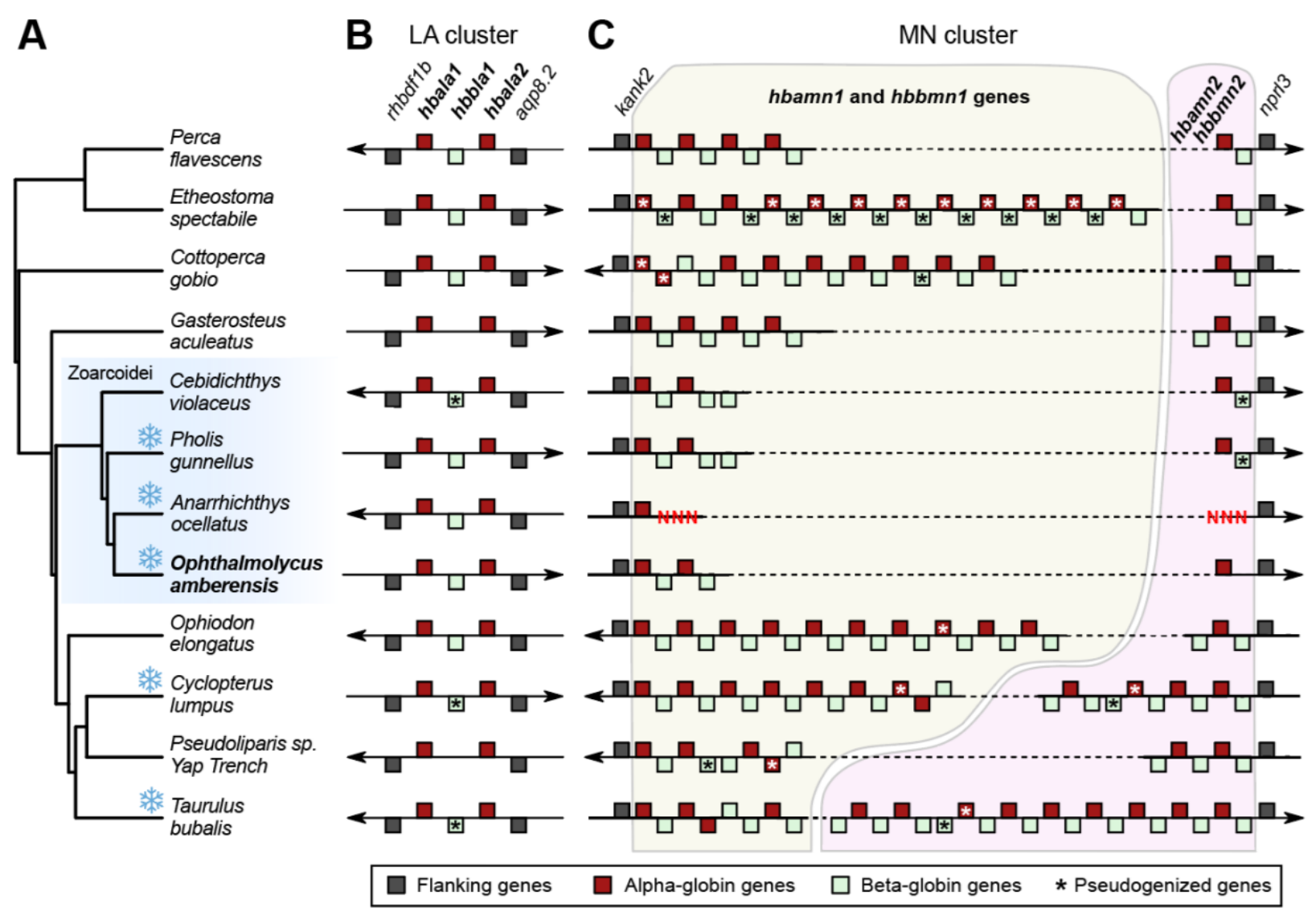

Figure 4. Evolution of the hemoglobin clusters in Perciformes. (A) The phylogenetic relationship of the studied perciform species was extracted from Rabosky et al. (2018). Snowflakes indicate species with distributions that overlap polar regions. (B) Genomic organization of the hemoglobin LA cluster flanked by the genes rhbdf1b and aqp8.2 in all studied species. (C) Genomic organization of the hemoglobin MN cluster flanked by the genes kank2 and nprl3 in all studied species. Genes flanking the clusters are represented by dark-grey squares, alpha-globin genes $(h b a)$ are represented with red squares, betaglobin genes $(h b b)$ are represented with light green squares, and pseudogenes are labelled with an asterisk $\left(^{*}\right)$. The arrowheads indicate the direction of the clusters in each assembly and the position of the square above or below the line denotes the position of the gene on the forward or reverse strand, respectively when the arrow points to the right, and the opposite when the arrow points to the left. "N" in A. ocellatus denote that the assembly contains gaps between exon1 of hbamn1 and nprl3, therefore preventing the study of this cluster in this species. Basic metrics for the genome assemblies included in this analysis are provided in Table S1.

The sialic acid synthase (nans) region is well-conserved across and beyond Zoarcoidei (Fig. 5a). For afpIII, we found no evidence of AFPs in G. aculeatus and C. violaceus (family Stichaeidae; Fig. 5b). Among the four species with copies of some portion of an afplll gene (either exon 1, exon 2, or complete copies of both exons 1 and 2 in order), copy number varied widely. Lycodicthys dearborni has at least 25 complete copies of afp/I/ with a number of partial copies (either exon 1 or exon 2 in isolation) scattered throughout the same region. Similarly, $O$. amberensis also had a high number of complete afplll copies (at least 13) with partial copies in the same region. Pholis gunnellus has at least 15 complete afp/ll copies as we all as partial copies. Finally, $A$. ocellatus has evidence of afp/l/ genes but with four complete afp/l/ copies spread over four of the six scaffolds including syntenic components of the afplll, it is unclear 
how many copies are truly present (Fig. 5b). This is primarily because, unlike L. dearborni for instance, no $A$. ocellatus scaffold has evidence of both afplll genes and flanking regions.

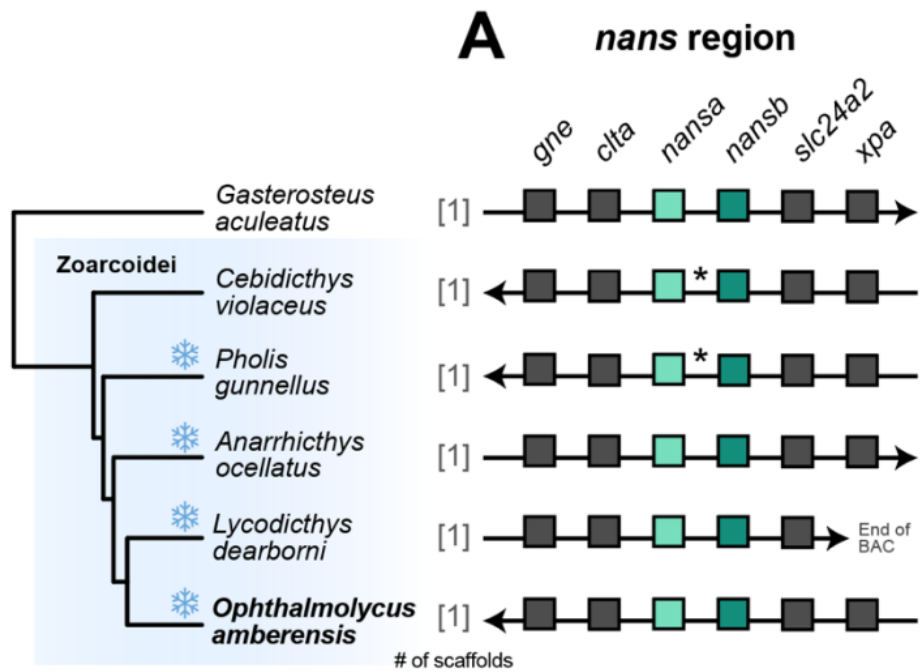

B

afpIII region

Figure 5. Synteny of the (A) sialic acid synthase (nans) and (B) afp/ll region in the suborder Zoarcoidei with three-spined stickleback (G. aculeatus) as the outgroup. Asterisks in (A) indicate evidence for additional nans gene copies in $C$. violaceus and $P$. gunnellus (1 and 8 extra copies, respectively). In (B), the $P$. gunnellus assembly has one scaffold that contains the afplll flanking genes as well as evidence of afpIII genes between them as well as a second scaffold that also has evidence of afp/ll genes. This is denoted by a second afp/ll region (red line) directly below the primary scaffold. Snowflakes indicate species with distributions that overlap polar regions and stars indicate breakpoints between scaffolds. As in Figure 4, arrowheads indicate the direction of the clusters in each assembly and the position of the square above or below the line denotes the position of the gene on the forward or reverse strand, respectively when the arrow points to the right, and the opposite when the arrow points to the left. Basic metrics for the genome assemblies included in this analysis (all species except for $L$. dearborni which is not a genome assembly) are provided in Table S1.

\section{Discussion:}

The rapid accumulation of genomic resources across the tree of life is empowering a new era of comparative genome science (Hotaling et al., in press; Marks, Hotaling, Frandsen, \& VanBuren, in press). Moreover, the rise of long-read sequencing has improved the contiguity of difficult to assemble, repeat-rich portions of genomes. Indeed, genome assemblies that integrate longreads are on average $48 x$ more contiguous than those that do not (Hotaling, Sproul, et al., 2021). In this study, we used long-reads to generate a highly contiguous assembly for an Antarctic eelpout, Ophthalmolycus amberensis, the first for the family Zoarcidae. We compared the genome of $O$. amberensis to other high-quality assemblies for polar and non-polar fishes with an emphasis on aspects that are difficult or impossible to assess with short-reads alone (repetitive elements and repeat-rich, dynamic loci underlying key phenotypes). Collectively, our results provide new understanding of adaptation to the extreme conditions of the Southern Ocean and highlight the power of long reads for new levels of genomic insight in non-model species.

\section{Repetitive element dynamics}

Repetitive elements (REs) are major drivers of genome evolution in fish and their abundance varies widely across species. Transposable elements (TEs) make up the largest fraction of REs in fish genomes ranging from 5-56\% (Shao, Han, \& Peng, 2019). Ophthalmolycus amberensis 
550

551

552

553

554

555

556

557

558

559

560

561

562

563

564

565

566

567

568

569

570

571

572

573

574

575

576

577

578

579

580

581

582

583

584

585

586

587

588

589

590

591

592

593

594

595

596

597

598

599

600

is intermediate among fish lineages with a genomic proportion of $~ 31 \%$, of which nearly $11 \%$ are classified as TEs. Our findings generally corroborate previous studies that show DNA transposons and LINEs are consistently abundant in fish genomes (e.g., Chalopin et al. 2015) and that RE abundance roughly correlates with genome size. The age distribution of TEs in $O$. amberensis revealed dynamic shifts in its TE landscape. While LINEs dominated present TE activity, DNA transposons and Helitrons both showed earlier bursts of activity.

Recent studies have highlighted a potential link between environmental stress and bursts of TE activity in fish (Chénais, Caruso, Hiard, \& Casse, 2012). In Antarctic notothenioid fish, DIRS1 retrotransposons form hotspots of chromosome rearrangements within lineages, potentially driving speciation (Auvinet et al., 2018; Auvinet et al., 2019). Heating and cooling cycles in polar seas have also been hypothesized to induce environmental stress which in turn drives bursts of TE activity (Auvinet et al., 2018). Consistent with this, we found evidence of recent major DIRS expansion in the notothenioid, C. aceratus, but not in other polar fish lineages (Fig. S1). And, more broadly, greater RE abundance and more evidence of historical activity bursts in the four polar fishes included in our analysis relative to the temperate fishes, despite their lack of phylogenetic (and spatial) overlap (Fig. 2, Fig. S1). It is possible that these signatures reflect historical, climate-induced shifts that affected fishes at both poles. However, this hypothesis is undermined by the fact that different fish groups adapted to cold environments at different times in history. Future efforts to integrate dense taxonomic sampling will allow for contextualizing links between TE activity, environmental stress, and genome evolution, particularly as they relate to ancient and contemporary environmental change.

\section{Selection on protein-coding genes}

Across thousands of shared orthologs, only a small fraction exhibited signatures of selection in either polar fish group (notothenioids or $O$. amberensis), and of those, just 10 genes were shared between the two. The split between Zoarcoidei and Notothenioidei occurred roughly 80 million years ago (Rabosky et al., 2018). Thus, with a moderate amount of shared ancestry, if adaptation to cold environments relies on universal biological and physiological mechanisms, we might expect a signature of parallel evolution in both groups evidenced by significant overlap between the targets of selection. This was not the case. However, among the subset that did overlap, two common themes emerged: selection has acted on genes involved in (i) membrane structure and (ii) DNA repair. Indeed, $50 \%$ of the shared genes have functional links to membrane structure in vertebrates. Membranes play an obvious role in organismal and cell survival as they delimit cellular structures, mediate transmembrane movement of solutes and other materials, and play key roles in many other key processes and structures (Rothfield, 2014). Moreover, because environmental conditions play a key role in determining how lipids-a key component of biological membranes-arrange themselves (Rothfield, 2014), it is unsurprising that membranes are important for thermal adaptation in a wide variety of organisms (Hazel, 1995). It is perhaps also unsurprising that fishes living in the most consistently cold marine habitats on Earth have experienced positive selection on genes related to membrane structure and function.

Generally speaking, membrane fluidity is maintained as temperature changes, a phenomenon known as homeoviscous adaptation (HVA; Hazel, 1995). However, the extreme, but stable, cold temperatures of polar seas means that maintenance of an HVA response to changing conditions should not hold any fitness benefit (Cossins \& Prosser, 1978). Still, polar fish membranes must function at low temperature and directional selection may have acted on this trait. Another target of selection in polar fishes, snx18, in concert with fip5, is involved in the morphogenesis of the epithelial lumen including the formation of membrane tubules (Willenborg et al., 2011). While the exact significance of selection on membrane-associated genes in polar 
601

602

603

604

605

606

607

608

609

610

611

612

613

614

615

616

617

618

619

620

621

622

623

624

625

626

627

628

629

630

631

632

633

634

635

636

637

638

639

640

641

642

643

644

645

646

647

648

649

650

651

fish remains to be determined, it is plausible that at least one major driver is functioning in the cold.

High amounts of dissolved oxygen in the Southern Ocean can boost the production of reactive oxygen species (ROS) in marine organisms (Pischedda et al., 2018). ROS damage cellular molecules including DNA (Van Houten, Santa-Gonzalez, \& Camargo, 2018). Intact and highfunctioning DNA repair pathways may be particularly crucial for the survival of Antarctic species. We found evidence for selection on two DNA repair-associated genes in polar fishes, $m s h 3$ and sprtn. A heterodimer between the proteins encoded by $m s h 3$ and a related gene, $m s h 2$, binds to the DNA helix and slides down it until mis-paired bases or other lesions are recognized (PećinaŠlaus, Kafka, Salamon, \& Bukovac, 2020). The other gene, sprtn, is a key component of the DNA replication machinery and is required for replication, thereby playing an essential role in the maintenance of genomic stability (Halder et al., 2019). While evidence of positive selection on DNA repair pathways has also been observed for deep sea fishes (Mu et al., 2021), and $O$. amberensis commonly occurs in cold and deep waters, the notothenioids included in our analysis are shallower water species. Our results suggest that evolution to mitigate elevated oxidative stress may have influenced polar fish evolution. In the case of $O$. amberensis, and other species living at depth in polar seas, a dual-benefit from selection on DNA repair pathways may be occurring.

\section{Evolution of hemoglobin genes in Zoarcoidei}

Hemoglobin is a major trait for adaptation to different environments in fishes (Powers, 1980; Verde, Giordano, di Prisco, \& Andersen, 2012). Because the capacity of hemoglobin to bind oxygen is sensitive to environmental conditions such as temperature and $\mathrm{pH}$, the molecular evolution of hemoglobin genes has been proposed to be necessary for fish to maintain respiratory homeostasis in new or changing environments (Powers, 1980). Vertebrate hemoglobin is a tetrameric protein composed of two alpha-globin and two beta-globin subunits. Combinations of different alpha and beta subunits can result in multiple hemoglobin tetramers with different functions. This "hemoglobin multiplicity" may also be a source of adaptive potential (Brix, Clements, \& Wells, 1999; Houston \& Gingras-Bedard, 1994; Verde et al., 2012). Hemoglobin multiplicity may be, at least in part, linked to hemoglobin gene copy number. For instance, cod fishes living in shallow and unstable environments have more hemoglobin gene copies than species living in more stable deep-sea environments (Baalsrud, Voje, et al., 2017). The Southern Ocean is a chronically cold and hyper-oxygenated environment, yet stable marine habitat. In this environment, the suborder Notothenioidei, which includes the white-blooded Antarctic icefishes lacking functional hemoglobin (Kim et al., 2019; Near et al., 2006), are the most common fish species. A reduction of hemoglobin multiplicity prior to cold adaptation followed by a decline in oxygen affinity has been documented in cold-adapted notothenioids (Verde, Parisi, \& di Prisco, 2006). In contrast, studies of hemoglobin evolution in other polar species, as well as other Zoarcoidei, remain scarce (di Prisco et al., 1990; Verde et al., 2002; Weber, Hourdez, Knowles, \& Lallier, 2003). Eelpouts and other species in the suborder Zoarcodei, however, inhabit diverse environments from pole to pole and from the surface to hadal depths, making them excellent models for understanding hemoglobin evolution across disparate environments.

Across ray-finned fishes in the order Perciformes living in a wide range of habitats, the two teleost hemoglobin clusters, LA and MN (Opazo, Butts, Nery, Storz, \& Hoffmann, 2013), have followed dramatically different evolutionary paths (Fig. 4). For the most part, the LA cluster has remained stable across species, with two alpha and one beta-globin genes (Opazo et al. 2013), and only a few instances of pseudogenization or loss of the beta-globin gene (Fig. 4a). In contrast, the MN cluster showed dramatic lineage-specific evolution, in line with other teleosts 
(Opazo et al., 2013). In most species in our comparison, the MN cluster expanded by multiple tandem duplications with frequent pseudogenization. In contrast, the genetic organization of the MN cluster has been relatively stable within Zoarcoidei, with three alpha-globin and two or three beta-globin genes (Fig. 4b). However, all studied Zoarcoidei share either the pseudogenization or loss of the hbbmn2 gene, which is predicted to be functional in all other perciformes included. Interestingly, all studied Zoarcoidei appear to have three to five hemoglobins (di Prisco et al., 1990; Verde et al., 2002; Weber et al., 2003) despite a relatively low number of hemoglobin genes in comparison to other perciformes. Thus, at least for Zoarcoidei, globin gene copy number does not seem to correlate with hemoglobin multiplicity. The relative stability of the Zoarcoidei MN cluster is also remarkable as the studied species inhabit similar environments to other species with highly variable MN clusters. This suggests that adaptation in Zoarcoidei hemoglobin may have been focused at the gene sequence level rather than duplication as seen in these other perciformes. The study of hemoglobin should be pursued in more Zoarcoidei species, including in the globally distributed eelpouts (Hotaling, Borowiec, et al., 2021), to better understand the molecular mechanisms underlying adaptation to environmental conditions.

\section{Evolution of antifreeze proteins}

The evolution of antifreeze proteins (AFP) is a hallmark of adaptation to cold environments across a wide range of taxa (Atıcı \& Nalbantoğlu, 2003; Davies et al., 2002). Multiple routes to AFP evolution have been documented in polar fishes, including the rise of afplll from neofunctionalization of a duplicated sialic acid synthase $b$ (nansb) gene in the Zoarcoidei (Deng et al., 2010) and the evolution of AFGPs in both notothenioids and Arctic cod (Chen et al., 1997a, 1997b; Zhuang et al., 2019) via two distinct molecular routes (Baalsrud, Tørresen, et al., 2017). Independent evolution of AFPs through non-overlapping pathways is one of the most well-known differences between the adaptation of eelpouts and notothenioids to the Southern Ocean. However, understanding of AFP evolution within the suborder Zoarcoidei-for which eelpouts comprise the most speciose family (Zoarcidae) - remains incomplete, largely due to the complexity of its history with many species and isoforms evolving at both poles and the complexity of the genomic region underlying it. Recent phylogenetic evidence suggests that afplll arose only once, early in the evolution of the Zoarcoidei approximately 18 million years ago (Hobbs et al., 2020). The lack of afpllI evidence in the genome of the monkeyface prickleback $C$. violaceus (family Stichaeidae) poses an additional question about the evolution of AFPs in Zoarcoidei. To our understanding, every previously surveyed species in the suborder has exhibited apflll evidence at the sequence level (at least 14 species from six families, Hobbs et al., 2020). Given that $C$. violaceus is part of the Zoarcoidei "in group" (Hotaling, Borowiec, et al., 2021) and is more closely related to other species in the suborder than the Alaskan ronquil Bathymaster caeruleofasciatus, which has afplll sequence evidence (Hobbs et al., 2020), its lack of apflll evidence at the sequence level suggests that either the afplll gene did not arise only once at the base of the clade, it has been horizontally transferred within the clade (e.g., Graham, Lougheed, Ewart, \& Davies, 2008), or most likely, it has been secondarily lost in C. violaceus. It is also possible that this finding could be due to assembly error, but this is unlikely given the assembly's high-quality (scaffold N50 $=6.72 \mathrm{Mb}$, Table S1), lack of N's in the predicted afp/ll region, and contiguity of flanking regions (Fig. 5).

Gene duplication, rearrangement, and modification has long obscured the study of AFP evolution (e.g., Deng et al., 2010). afplll copy number varies considerably between closely related species (Scott, Fletcher, \& Davies, 1986) and even within species across their geographic range (Hew et al., 1988). Short-read genome assemblies limit afpll/ copy number inference. For instance, the recent short-read assembly of $A$. ocellatus has afp/l/ sequences on four different scaffolds which are all $\sim 2 \mathrm{~kb}$ in length with identical sequences (including for afpllI genes). However, long-read assemblies can largely ameliorate this issue. Indeed, following the 
703

704

705

706

707

708

709

710

711

712

713

714

715

716

717

718

719

720

721

722

723

724

725

726

727

728

729

730

731

732

733

734

735

736

737

738

739

740

741

742

743

744

745

746

747

748

749

750

751

752

753

massive improvements of long-read sequencing for genome assembly, a complete sequence of the complex afp/ll genome has now been produced (for $P$. gunnellus) with a second, near complete sequence reported in this study. For both Antarctic eelpouts, $O$. amberensis and $L$. dearborni, and the rock gunnell, $P$. gunnellus, a large number of similar but not identical afpIII copies (at least 13, 25, and 15 respectively, Fig. 5) may represent an evolutionarily tuned system for depressing the serum freezing point (Kelley, Aagaard, MacCoss, \& Swanson, 2010).

\section{Adaptation to the Southern Ocean: new insights from long-read sequencing}

We have entered an exciting time for genome biology. It is now possible to characterize complex regions of genomes to better understand evolution across virtually any group of interest. In this study, we used long-read sequencing to generate a high-quality reference assembly for an Antarctic eelpout, O. amberensis, the first for the group. We used this new genomic resource to better understand the evolution of $O$. amberensis in the Southern Ocean, particularly as it relates to other Antarctic fish specialists (i.e., notothenioids) and the rest of the suborder Zoarcoidei. We showed evidence of historical shifts in TE activity in O. amberensis and other polar fishes, perhaps reflecting an ancient burst of TE activity in response to environmental change. Moreover, we found little overlap between the targets of natural selection in $O$. amberensis and in notothenioids inhabiting the same habitats, indicating that adaptation of both groups to the Southern Ocean appear to have followed largely different trajectories. However, we did find overlap in a few targets of selection, many of which were genes involved in DNA repair or membrane structure. We also observed the relative evolutionary stability of the hemoglobin MN cluster in the Zoarcoidei relative to other teleosts despite these species inhabiting similar environments to species with much more diverse MN clusters. For AFPs, we identified the first species in the suborder Zoarcoidei with no evidence of afp/ll genes (C. violaceus), likely reflecting a lineage-specific loss of the cluster. To further understand the evolution of polar fishes, both within and across groups, it is clear that more dense taxonomic sampling is needed. Snailfishes (order Scorpaeniformes; family Liparidae) occur around the world, often at hadal depths, and are the most species-rich deep-water family in the Southern Ocean, making them another exceptional candidate for comparisons to understand the general rules of Antarctic adaptation.

Beyond polar fish adaptation, the utility of long-read sequencing for understanding complex regions of genomes is clear. The power of these data will increase as accuracy continues to improve (e.g. circular consensus sequencing (i.e., PacBio HiFi, Wenger et al., 2019)) and reads lengthen. The application of long-read technologies to eelpouts and other zoarcids will yield additional high-quality, contiguous sequences of the afp/ll locus. The limiting factor for understanding questions similar to those posed in this study has likely shifted from generating high-quality assemblies to obtaining samples compatible with high-molecular weight DNA extraction and long-read sequencing. We echo previous studies (e.g., Buckner, Sanders, Faircloth, \& Chakrabarty, 2021; Hotaling et al., in press) that the field needs curated specimen vouchers with properly stored tissues and associated metadata to empower the future of genome biology in polar fishes and beyond.

\section{Acknowledgements:}

We thank the captain and crew of the ARSV Laurence M. Gould, the personnel of the U.S. Antarctic Program Support Contractors for assistance in Chile, at sea, and at Palmer Station, as well as the logistics in Denver, $\mathrm{CO}$ for their support related to the Antarctic fieldwork required for this study. We also thank Blair Perry, Kerry McGowan, and Ellie Armstrong for comments that improved the manuscript. We acknowledge funding from NSF awards OPP-1543383 and OPP1947040 (supporting T.D.), and OPP-1906015 (to J.L.K.) as well as funding from the Antarctic Science Bursary. The RNA sequencing data used in this study was provided as part of a 
754

755

756

757

758

759

760

761

762

763

764

765

766

767

768

769

770

771

772

773

774

775

776

777

778

779

780

781

782

783

784

785

786

787

788

789

790

791

792

793

794

795

796

797

798

799

800

801

802

803

804

workshop sponsored by the NSF (OPP-1744877) and a Grant in Aid of Research through Long Island University-Post (both to Scott Santagata). We acknowledge computing resources from the Center for Institutional Research Computing at Washington State University and the NSF Extreme Science and Engineering Discovery Environment (XSEDE).

\section{References:}

Ahn, D.-H., Shin, S. C., Kim, B.-M., Kang, S., Kim, J.-H., Ahn, I., . . Park, H. (2017). Draft genome of the Antarctic dragonfish, Parachaenichthys charcoti. GigaScience, 6(8), gix060.

Akbarzadeh, A., Günther, O. P., Houde, A. L., Li, S., Ming, T. J., Jeffries, K. M., .. Miller, K. M. (2018). Developing specific molecular biomarkers for thermal stress in salmonids. BMC Genomics, 19(1), 1-28.

Anderson, M. E. (1994). Systematics and osteology of the Zoarcidae (Teleostei: Perciformes). Ichthyological Bulletin of the J.L.B. Smith Institute of Ichthyology, 60, 1-120.

Andrews, S. (2010). FastQC: a quality control tool for high throughput sequence data.

Atıcı, Ö., \& Nalbantoğlu, B. (2003). Antifreeze proteins in higher plants. Phytochemistry, 64(7), 1187-1196.

Auvinet, J., Graça, P., Belkadi, L., Petit, L., Bonnivard, E., Dettaï, A., . . Higuet, D. (2018). Mobilization of retrotransposons as a cause of chromosomal diversification and rapid speciation: the case for the Antarctic teleost genus Trematomus. BMC Genomics, 19(1), $1-18$.

Auvinet, J., Graça, P., Ghigliotti, L., Pisano, E., Dettaï, A., Ozouf-Costaz, C., \& Higuet, D. (2019). Insertion hot spots of DIRS1 Retrotransposon and chromosomal diversifications among the Antarctic Teleosts Nototheniidae. International journal of molecular sciences, 20(3), 701.

Baalsrud, H. T., Tørresen, O. K., Solbakken, M. H., Salzburger, W., Hanel, R., Jakobsen, K. S., \& Jentoft, S. (2017). De novo gene evolution of antifreeze glycoproteins in codfishes revealed by whole genome sequence data. Mol Biol Evol, 35(3), 593-606.

Baalsrud, H. T., Voje, K. L., Tørresen, O. K., Solbakken, M. H., Matschiner, M., Malmstrøm, M., ... Jentoft, S. (2017). Evolution of hemoglobin genes in codfishes influenced by ocean depth. Sci Rep, 7(1), 1-10.

Beck, E. A., Healey, H. M., Small, C. M., Currey, M. C., Desvignes, T., Cresko, W. A., \& Postlethwait, J. H. (2021). Advancing human disease research with fish evolutionary mutant models. Trends in Genetics.

Benjamini, Y., \& Hochberg, Y. (1995). Controlling the false discovery rate: a practical and powerful approach to multiple testing. Journal of the Royal statistical society: series $B$ (Methodological), 57(1), 289-300.

Bista, I., McCarthy, S. A., Wood, J., Ning, Z., Detrich lii, H. W., Desvignes, T., . . Torrance, J. (2020). The genome sequence of the channel bull blenny, Cottoperca gobio (Günther, 1861). Wellcome Open Research, 5.

Bradnam, K. R., Fass, J. N., Alexandrov, A., Baranay, P., Bechner, M., Birol, I., . . Chikhi, R. (2013). Assemblathon 2: evaluating de novo methods of genome assembly in three vertebrate species. GigaScience, 2(1), 2047-2217X-2042-2010.

Brix, O., Clements, K., \& Wells, R. (1999). Haemoglobin components and oxygen transport in relation to habitat distribution in triplefin fishes (Tripterygiidae). Journal of Comparative Physiology B, 169(4), 329-334.

Buckner, J. C., Sanders, R. C., Faircloth, B. C., \& Chakrabarty, P. (2021). Science Forum: The critical importance of vouchers in genomics. Elife, 10, e68264.

Chen, L., DeVries, A. L., \& Cheng, C.-H. C. (1997a). Convergent evolution of antifreeze glycoproteins in Antarctic notothenioid fish and Arctic cod. Proceedings of the National Academy of Sciences, 94(8), 3817-3822. 
805

806

807

808

809

810

811

812

813

814

815

816

817

818

819

820

821

822

823

824

825

826

827

828

829

830

831

832

833

834

835

836

837

838

839

840

841

842

843

844

845

846

847

848

849

850

851

852

853

854

Chen, L., DeVries, A. L., \& Cheng, C.-H. C. (1997b). Evolution of antifreeze glycoprotein gene from a trypsinogen gene in Antarctic notothenioid fish. Proceedings of the National Academy of Sciences, 94(8), 3811-3816. doi:10.1073/pnas.94.8.3811

Chen, L., Lu, Y., Li, W., Ren, Y., Yu, M., Jiang, S., . . Bilyk, K. T. (2019). The genomic basis for colonizing the freezing Southern Ocean revealed by Antarctic toothfish and Patagonian robalo genomes. GigaScience, 8(4), giz016.

Chénais, B., Caruso, A., Hiard, S., \& Casse, N. (2012). The impact of transposable elements on eukaryotic genomes: from genome size increase to genetic adaptation to stressful environments. Gene, 509(1), 7-15.

Cossins, A., \& Prosser, C. (1978). Evolutionary adaptation of membranes to temperature. Proceedings of the National Academy of Sciences, 75(4), 2040-2043.

Davies, P. L., Baardsnes, J., Kuiper, M. J., \& Walker, V. K. (2002). Structure and function of antifreeze proteins. Philos Trans R Soc Lond B Biol Sci, 357(1423), 927-935. doi:10.1098/rstb.2002.1081

Deng, C., Cheng, C. H., Ye, H., He, X., \& Chen, L. (2010). Evolution of an antifreeze protein by neofunctionalization under escape from adaptive conflict. Proc Natl Acad Sci U S A, 107(50), 21593-21598. doi:10.1073/pnas.1007883107

DeVries, A. L., \& Steffensen, J. F. (2005). The Arctic and Antarctic polar marine environments. Fish Physiology, 22, 1-24. doi:10.1016/S1546-5098(04)22001-5

di Prisco, G., D'Avino, R., Camardella, L., Caruso, C., Romano, M., \& Rutigliano, B. (1990). Structure and function of hemoglobin in Antarctic fishes and evolutionary implications. Polar Biology, 10(4), 269-274.

Feron, R., Zahm, M., Cabau, C., Klopp, C., Roques, C., Bouchez, O., . . Haffray, P. (2020). Characterization of a Y-specific duplication/insertion of the anti-Mullerian hormone type II receptor gene based on a chromosome-scale genome assembly of yellow perch, Perca flavescens. Molecular Ecology Resources, 20(2), 531-543.

Flynn, J. M., Hubley, R., Goubert, C., Rosen, J., Clark, A. G., Feschotte, C., \& Smit, A. F. (2020). RepeatModeler2 for automated genomic discovery of transposable element families. Proceedings of the National Academy of Sciences, 117(17), 9451-9457.

Ge, S. X., Jung, D., \& Yao, R. (2020). ShinyGO: a graphical gene-set enrichment tool for animals and plants. Bioinformatics, 36(8), 2628-2629.

Graham, L. A., Lougheed, S. C., Ewart, K. V., \& Davies, P. L. (2008). Lateral transfer of a lectinlike antifreeze protein gene in fishes. PLoS One, 3(7), e2616. doi:10.1371/journal.pone.0002616

Griffiths, H. J. (2010). Antarctic marine biodiversity-what do we know about the distribution of life in the Southern Ocean? PLoS One, 5(8), e11683.

Halder, S., Torrecilla, I., Burkhalter, M. D., Popović, M., Fielden, J., Vaz, B., . . Wiseman, K. (2019). SPRTN protease and checkpoint kinase 1 cross-activation loop safeguards DNA replication. Nat Commun, 10(1), 1-18.

Hazel, J. R. (1995). Thermal adaptation in biological membranes: is homeoviscous adaptation the explanation? Annual Review of Physiology, 57(1), 19-42.

Heras, J., Chakraborty, M., Emerson, J., \& German, D. P. (2020). Genomic and biochemical evidence of dietary adaptation in a marine herbivorous fish. Proceedings of the Royal Society B, 287(1921), 20192327.

Hew, C. L., Wang, N., Joshi, S., Fletcher, G., Scott, G., Hayes, P., . . Davies, P. (1988). Multiple genes provide the basis for antifreeze protein diversity and dosage in the ocean pout, Macrozoarces americanus. Journal of Biological Chemistry, 263(24), 12049-12055.

Hobbs, R. S., Hall, J. R., Graham, L. A., Davies, P. L., \& Fletcher, G. L. (2020). Antifreeze protein dispersion in eelpouts and related fishes reveals migration and climate alteration within the last 20 Ma. PLoS One, 15(12), e0243273. doi:10.1371/journal.pone.0243273 
855

856

857

858

859

860

861

862

863

864

865

866

867

868

869

870

871

872

873

874

875

876

877

878

879

880

881

882

883

884

885

886

887

888

889

890

891

892

893

894

895

896

897

898

899

900

901

902

903

904

905

Holt, C., \& Yandell, M. (2011). MAKER2: an annotation pipeline and genome-database management tool for second-generation genome projects. BMC Bioinformatics, 12, 491. doi:10.1186/1471-2105-12-491

Hotaling, S., Borowiec, M. L., Lins, L. S., Desvignes, T., \& Kelley, J. L. (2021). The biogeographic history of eelpouts and related fishes: linking phylogeny, environmental change, and patterns of dispersal in a globally distributed fish group. Molecular phylogenetics and evolution, 107211.

Hotaling, S., Kelley, J. L., \& Frandsen, P. B. (in press). Towards a genome sequence for every animal: where are we now? Proceedings of the National Academy of Sciences.

Hotaling, S., Sproul, J., Heckenhauer, J., Powell, A., Larracuente, A., Pauls, S., . . Frandsen, P. (2021). Long-reads are revolutionizing 20 years of insect genome sequencing. Genome Biology and Evolution, evab138.

Houston, A., \& Gingras-Bedard, J. H. (1994). Variable versus constant temperature acclimation regimes: effects on hemoglobin isomorph profile in goldfish, Carassius auratus. Fish physiology and biochemistry, 13(6), 445-450.

Kelley, J. L., Aagaard, J. E., MacCoss, M. J., \& Swanson, W. J. (2010). Functional diversification and evolution of antifreeze proteins in the antarctic fish Lycodichthys dearborni. J Mol Evol, 71(2), 111-118. doi:10.1007/s00239-010-9367-6

Kim, B.-M., Amores, A., Kang, S., Ahn, D.-H., Kim, J.-H., Kim, I.-C., . . Lee, J. (2019). Antarctic blackfin icefish genome reveals adaptations to extreme environments. Nature Ecology \& Evolution, 3(3), 469-478.

Koren, S., Walenz, B. P., Berlin, K., Miller, J. R., Bergman, N. H., \& Phillippy, A. M. (2017). Canu: scalable and accurate long-read assembly via adaptive k-mer weighting and repeat separation. Genome research, 27(5), 722-736.

Korf, I. (2004). Gene finding in novel genomes. BMC Bioinformatics, 5(1), 1-9.

Krueger, F. (2015). Trim Galore!: a wrapper tool around Cutadapt and FastQC to consistently apply quality and adapter trimming to FastQ files. Babraham Bioinformatics, Cambridge, United Kingdom. In.

$\mathrm{Li}, \mathrm{H}$. (2013). Aligning sequence reads, clone sequences and assembly contigs with BWA-MEM. arXiv, 1303.3997.

Li, H., Handsaker, B., Wysoker, A., Fennell, T., Ruan, J., Homer, N., . . Durbin, R. (2009). The sequence alignment/map format and SAMtools. Bioinformatics, 25(16), 2078-2079.

Li, X., Trinh, K.-Y., Hew, C. L., Buettner, B., Baenziger, J., \& Davies, P. L. (1985). Structure of an antifreeze polypeptide and its precursor from the ocean pout, Macrozoarces americanus. Journal of Biological Chemistry, 260(24), 12904-12909.

Longo, G. C., Lam, L., Basnett, B., Samhouri, J., Hamilton, S., Andrews, K., ... Nichols, K. M. (2020). Strong population differentiation in lingcod (Ophiodon elongatus) is driven by a small portion of the genome. Evol Appl, 13(10), 2536-2554.

Löytynoja, A. (2014). Phylogeny-aware alignment with PRANK. In Multiple sequence alignment methods (pp. 155-170): Springer.

Marks, R. A., Hotaling, S., Frandsen, P. B., \& VanBuren, R. (in press). Representation and participation across 20 years of plant genome sequencing. Nature Plants.

Melsted, P., \& Pritchard, J. K. (2011). Efficient counting of k-mers in DNA sequences using a bloom filter. BMC Bioinformatics, 12(1), 1-7.

Møller, P. R., Nielsen, J. G., \& Anderson, M. E. (2005). Systematics of polar fishes. Fish Physiology, 22, 25-78. doi:10.1016/S1546-5098(04)22002-7

Moore, J. K., Abbott, M. R., \& Richman, J. G. (1999). Location and dynamics of the Antarctic Polar Front from satellite sea surface temperature data. Journal of Geophysical Research: Oceans, 104(C2), 3059-3073.

Moran, A. L., \& Woods, H. A. (2012). Why might they be giants? Towards an understanding of polar gigantism. Journal of Experimental Biology, 215(12), 1995-2002. 
906

907

908

909

910

911

912

913

914

915

916

917

918

919

920

921

922

923

924

925

926

927

928

929

930

931

932

933

934

935

936

937

938

939

940

941

942

943

944

945

946

947

948

949

950

951

952

953

954

955

956
Moran, R. L., Catchen, J. M., \& Fuller, R. C. (2020). Genomic resources for darters (Percidae: Etheostominae) provide insight into postzygotic barriers implicated in speciation. Mol Biol Evol, 37(3), 711-729.

Mu, Y., Bian, C., Liu, R., Wang, Y., Shao, G., Li, J., . . Ao, J. (2021). Whole genome sequencing of a snailfish from the Yap Trench $(\sim 7,000 \mathrm{~m})$ clarifies the molecular mechanisms underlying adaptation to the deep sea. PLoS Genet, 17(5), e1009530.

Near, T. J., Dornburg, A., Kuhn, K. L., Eastman, J. T., Pennington, J. N., Patarnello, T., . . . Jones, C. D. (2012). Ancient climate change, antifreeze, and the evolutionary diversification of Antarctic fishes. Proceedings of the National Academy of Sciences, 109(9), 3434-3439. doi:10.1073/pnas.1115169109

Near, T. J., Parker, S. K., \& Detrich III, H. W. (2006). A genomic fossil reveals key steps in hemoglobin loss by the antarctic icefishes. Mol Biol Evol, 23(11), 2008-2016.

Opazo, J. C., Butts, G. T., Nery, M. F., Storz, J. F., \& Hoffmann, F. G. (2013). Whole-genome duplication and the functional diversification of teleost fish hemoglobins. Mol Biol Evol, 30(1), 140-153.

Pećina-Šlaus, N., Kafka, A., Salamon, I., \& Bukovac, A. (2020). Mismatch repair pathway, genome stability and cancer. Frontiers in molecular biosciences, 7, 122.

Pischedda, A., Ramasamy, K. P., Mangiagalli, M., Chiappori, F., Milanesi, L., Miceli, C., . . . Lotti, M. (2018). Antarctic marine ciliates under stress: superoxide dismutases from the psychrophilic Euplotes focardii are cold-active yet heat tolerant enzymes. Sci Rep, 8(1), 1-13.

Powers, D. A. (1980). Molecular ecology of teleost fish hemoglobins: strategies for adapting to changing environments. American Zoologist, 139-162.

R Core Team. (2021). R: A language and environment for statistical computing.

Rabosky, D. L., Chang, J., Title, P. O., Cowman, P. F., Sallan, L., Friedman, M., . . Coll, M. (2018). An inverse latitudinal gradient in speciation rate for marine fishes. Nature, 559(7714), 392-395. doi:10.1038/s41586-018-0273-1

Rice, P., Longden, I., \& Bleasby, A. (2000). EMBOSS: the European molecular biology open software suite. Trends in Genetics, 16(6), 276-277.

Robinson, J. T., Thorvaldsdóttir, H., Winckler, W., Guttman, M., Lander, E. S., Getz, G., \& Mesirov, J. P. (2011). Integrative genomics viewer. Nature Biotechnology, 29(1), 24-26.

Rothfield, L. I. (2014). Structure and function of biological membranes: Academic Press.

Ruud, J. T. (1954). Vertebrates without erythrocytes and blood pigment. Nature, 173(4410), 848-850.

Scott, G. K., Fletcher, G. L., \& Davies, P. L. (1986). Fish Antifreeze Proteins: Recent Gene Evolution. Canadian Journal of Fisheries and Aquatic Sciences, 43(5), 1028-1034. doi:10.1139/f86-128

Scott, G. K., Hew, C. L., \& Davies, P. L. (1985). Antifreeze protein genes are tandemly linked and clustered in the genome of the winter flounder. Proc Natl Acad Sci U S A, 82(9), 2613-2617.

Shao, F., Han, M., \& Peng, Z. (2019). Evolution and diversity of transposable elements in fish genomes. Sci Rep, 9(1), 1-8.

Sievers, F., Wilm, A., Dineen, D., Gibson, T. J., Karplus, K., Li, W., . . Söding, J. (2011). Fast, scalable generation of high-quality protein multiple sequence alignments using Clustal Omega. Molecular systems biology, 7(1), 539.

Simão, F. A., Waterhouse, R. M., loannidis, P., Kriventseva, E. V., \& Zdobnov, E. M. (2015). BUSCO: assessing genome assembly and annotation completeness with single-copy orthologs. Bioinformatics, 31(19), 3210-3212.

Simpson, J. T., \& Durbin, R. (2010). Efficient construction of an assembly string graph using the FM-index. Bioinformatics, 26(12), i367-373. doi:10.1093/bioinformatics/btq217

Smit, A., Hubley, R., \& Green, P. (2015). RepeatMasker Open-4.0. 2013-2015. In. 
957

958

959

960

961

962

963

964

965

966

967

968

969

970

971

972

973

974

975

976

977

978

979

980

981

982

983

984

985

986

987

988

989

990

991

992

993

994

995

996

997

998

999

1000

1001

1002

1003

1004

Smit, A. F., \& Hubley, R. (2008). RepeatModeler Open-1.0. In.

Stamatakis, A. (2014). RAxML version 8: a tool for phylogenetic analysis and post-analysis of large phylogenies. Bioinformatics, 30(9), 1312-1313. doi:10.1093/bioinformatics/btu033

Stanke, M., \& Waack, S. (2003). Gene prediction with a hidden Markov model and a new intron submodel. Bioinformatics, 19(suppl_2), ii215-ii225.

Van Houten, B., Santa-Gonzalez, G. A., \& Camargo, M. (2018). DNA repair after oxidative stress: current challenges. Current opinion in toxicology, 7, 9-16.

Verde, C., Carratore, V., Riccio, A., Tamburrini, M., Parisi, E., \& Di Prisco, G. (2002). The functionally distinct hemoglobins of the Arctic spotted wolffish Anarhichas minor. Journal of Biological Chemistry, 277(39), 36312-36320.

Verde, C., Giordano, D., di Prisco, G., \& Andersen, Ø. (2012). The haemoglobins of polar fish: evolutionary and physiological significance of multiplicity in Arctic fish. Biodiversity, 13(34), 228-233.

Verde, C., Parisi, E., \& di Prisco, G. (2006). The evolution of thermal adaptation in polar fish. Gene, 385, 137-145.

Vurture, G. W., Sedlazeck, F. J., Nattestad, M., Underwood, C. J., Fang, H., Gurtowski, J., \& Schatz, M. C. (2017). GenomeScope: fast reference-free genome profiling from short reads. Bioinformatics, 33(14), 2202-2204.

Weber, R. E., Hourdez, S., Knowles, F., \& Lallier, F. (2003). Hemoglobin function in deep-sea and hydrothermal-vent endemic fish: Symenchelis parasitica (Anguillidae) and Thermarces cerberus (Zoarcidae). Journal of Experimental Biology, 206(15), 2693-2702.

Wenger, A. M., Peluso, P., Rowell, W. J., Chang, P.-C., Hall, R. J., Concepcion, G. T., . . . Olson, N. D. (2019). Accurate circular consensus long-read sequencing improves variant detection and assembly of a human genome. Nature Biotechnology, 37(10), 1155-1162.

Willenborg, C., Jing, J., Wu, C., Matern, H., Schaack, J., Burden, J., \& Prekeris, R. (2011). Interaction between FIP5 and SNX18 regulates epithelial lumen formation. Journal of Cell Biology, 195(1), 71-86.

Xu, L., Dong, Z., Fang, L., Luo, Y., Wei, Z., Guo, H., .. X Xia, Q. (2019). OrthoVenn2: a web server for whole-genome comparison and annotation of orthologous clusters across multiple species. Nucleic Acids Res, 47(W1), W52-W58.

Xu, S., Wang, J., Guo, Z., He, Z., \& Shi, S. (2020). Genomic convergence in the adaptation to extreme environments. Plant communications, 100117.

Yang, Z. (2007). PAML 4: phylogenetic analysis by maximum likelihood. Mol Biol Evol, 24(8), 1586-1591.

Zhuang, X., Yang, C., Murphy, K. R., \& Cheng, C.-H. C. (2019). Molecular mechanism and history of non-sense to sense evolution of antifreeze glycoprotein gene in northern gadids. Proceedings of the National Academy of Sciences, 116(10), 4400-4405.

\section{Data accessibility:}

The genome assembly, raw sequence data, and associated files for $O$. amberensis are deposited under NCBI BioProject \#PRJNA701078 and this study's GitHub (http://github.io/oamberensis genome).

\section{Author contributions:}

S.H. and J.L.K. conceived of the study. L.S.F.L. performed laboratory work. S.H., T.D., J.S.S. and J.L.K. performed analyses. S.H. led the writing of the manuscript with considerable help from T.D., J.S.S., and J.L.K. T.D. provided the O. amberensis samples. All authors read and approved the final version of the manuscript. 